\title{
RELATIVE ENTROPY IN HYPERBOLIC RELAXATION FOR BALANCE LAWS*
}

\author{
ALEXEY MIROSHNIKOV ${ }^{\dagger}$ AND KONSTANTINA TRIVISA ${ }^{\ddagger}$
}

\begin{abstract}
We present a general framework for the approximation of systems of hyperbolic balance laws. The novelty of the analysis lies in the construction of suitable relaxation systems and the derivation of a delicate estimate on the relative entropy. We provide a direct proof of convergence in the smooth regime for a wide class of physical systems. We present results for systems arising in materials science, where the presence of source terms presents a number of additional challenges and requires delicate treatment. Our analysis is in the spirit of the framework introduced by Tzavaras [A. Tzavaras, Commun. Math. Sci., 3(2), 119-132, 2005] for systems of hyperbolic conservation laws.
\end{abstract}

Key words. Hypebolic relaxation, balance laws, relative entropy, weak dissipation.

AMS subject classifications. 35165, 76N10, 35B 45.

\section{Introduction}

We present a general framework for the approximation of systems of hyperbolic balance laws,

$$
\partial_{t} u+\partial_{\alpha} f_{\alpha}(u)=g(u) \quad u(x, t) \in \mathbb{R}^{n}, x \in \mathbb{R}^{d},
$$

by relaxation systems presented in the form of the extended system

$$
\partial_{t} U+\partial_{\alpha} F_{\alpha}(U)=\frac{1}{\varepsilon} R(U)+G(U), \quad U(x, t) \in \mathbb{R}^{N}, x \in \mathbb{R}^{d}
$$

in the regime where the solution of the limiting system (as $\varepsilon \rightarrow 0$ ) is smooth. Motivated by the structure of physical models and the analysis in [6], we deal with relaxation systems of type (1.2) which are equipped with a globally defined, convex entropy $H(U)$ satisfying

$$
\partial_{t} H(U)+\partial_{\alpha} Q_{\alpha}(U)=\frac{1}{\varepsilon} \mathrm{D} H(U) R(U)+\mathrm{D} H(U) G(U) .
$$

The problem of numerical approximation of nonlinear hyperbolic balance laws is extremely challenging. In the present article we identify a class of relaxation schemes suitable for the approximation of solutions to certain systems of hyperbolic balance laws arising in continuum physics. The relaxation schemes proposed in our work provide a very effective mechanism for the approximation of the solutions of these systems with a very high degree of accuracy.

The main contribution of the present article to the existing theory can be characterized as follows:

- This work provides a general framework describing how, given a physical system governed by a hyperbolic balance law, one can construct an extended

*Received: March 13, 2013; accepted (in revised form): July 16, 2013. Communicated by Francois Bouchut.

${ }^{\dagger}$ Department of Mathematics, University of Massachusetts, Amherst, MA 01003, USA (miroshnikov@math.umass.edu).

${ }^{\ddagger}$ Department of Mathematics, University of Maryland, College Park, MD 20742, USA (trivisa@ math.umd.edu). 
system endowed with a globally defined, convex entropy $H(U)$ and the resulting relaxation system for its approximation. It has the potential of being of use for the construction of suitable approximating schemes for a variety of hyperbolic balance laws. Our analysis treats a large class of physical systems such as the system of elasticity (6.1) (cf. Section 6), two phase flow models (7.2) (cf. Section 7), and general symmetric hyperbolic systems (8.1) (cf. Section 8). In the latter application the relaxation of the hyperbolic system is obtained by relaxing $n$-vector flux components.

- Our framework is applicable in the multidimensional setting and provides a rigorous proof of the relaxation limit and a rate of convergence for a large class of physically relevant hyperbolic balance laws. As is well known, results for multidimensional systems of hyperbolic balance laws are limited in the literature. In addition, our analysis treats a large class of source terms: those satisfying a special mechanism that induces dissipation as well as a more general source term.

We establish convergence of weak solutions of (1.2) to solutions of the equilibrium system (1.1) via a relative entropy argument which relies on (1.3). The proof provides a rate of convergence. The relative entropy method relies on the "weak-strong" uniqueness principle established by Dafermos for systems of conservation laws admitting convex entropy functional [9]; see also DiPerna [13]. In addition to the pioneering work of Dafermos and DiPerna, the relative entropy method has been successfully used to study hydrodynamic limits of particle systems [4, 14, 22, 21, 25], hydrodynamic limits from kinetic equations to multidimensional macroscopic models $[1,3,17]$, and the convergence of numerical schemes in the context of three-dimensional polyconvex elasticity $[18,20]$.

The main ingredients of our approach can be formulated as follows:

- A relative entropy inequality which provides a simple and direct convergence framework before formation of shocks. The reader may contrast the present framework to the classic convergence framework for relaxation limits, which proceeds through analysis of the linearized (collision or relaxation) operator $[26]$.

- Physically grounded structural hypotheses imposed on the relaxation system. These structural hypotheses will be of use for the derivation of the relative entropy inequality and for the proof of the desired convergence. The relative entropy computation hinges on entropy consistency [23], that is, the restriction of the entropy pair $H-Q_{\alpha}$ on the manifold of Maxwellians

$$
\mathcal{M}:=\left\{U \in \mathbb{R}^{N}: R(U)=0\right\}=\left\{U \in \mathbb{R}^{N}: U=M(u), u \in \mathbb{R}^{n}\right\}
$$

induces an entropy pair for the equilibrium system (1.1) in the form

$$
\eta(u)=H(M(u)), \quad q_{\alpha}=Q_{\alpha}(M(u)) .
$$

- A physically motivated dissipation mechanism (in the sense of (H8)) associated with the source term in (1.2) with respect to the manifold of Maxwellians on which relaxation takes place. The dissipation mechanism on (1.2) induces weak dissipation on the equilibrium balance law (1.1) due to source consistency requirement (cf. Section 2.4). The concept of weak dissipation for hyperbolic balance laws was introduced by Dafermos in [11]. To realize the role of dissipation in the present context, the reader may contrast the result 
of Theorem 3.1 for weakly dissipative source terms with Theorem 3.2 which corresponds to the case of a general source.

The paper is organized as follows: In Section 2 we present the structural hypotheses on (1.2) which are of use in the derivation of the relative entropy inequality and the proof of the desired convergence. In Section 3 we present the main theorems of this article for two different classes of source terms. In Section 4 we define the concept of relative entropy $H^{r}\left(U^{\varepsilon}, M(\bar{u})\right)$ and entropy fluxes $Q_{\alpha}^{r}\left(U^{\varepsilon}, M(\bar{u})\right)$. Section 5 contains the proof of the main result, which is based on error estimates for the approximation of the conserved quantities by the solution of the relaxation system. Applications to nonlinear elasticity and two phase flow models (combustion) are presented in Section 6 and Section 7, respectively. Finally, Section 8 provides a general framework describing how, given a physical system governed by a symmetric hyperbolic balance law, one can construct an extended system and the resulting relaxation system for its approximation.

\section{Notation and hypotheses}

For the convenience of the reader we collect in this section all the relevant notation and hypotheses. Here and in what follows:

(1) $G, R$, and $F_{\alpha}, \alpha=1, \ldots, d$ denote the mappings $G, R, F_{\alpha}: \mathbb{R}^{N} \rightarrow \mathbb{R}^{N}$, whereas $g$ and $f$ denote the maps $g, f: \mathbb{R}^{n} \rightarrow \mathbb{R}^{n}$. In our presentation, $G(U), R(U), F_{\alpha}(U), g(u)$ and $f(u)$ are treated as column vectors.

(2) $\mathrm{D}, \mathrm{D}_{u}$ denote the differentials with respect to the state vectors $U \in \mathbb{R}^{N}$ and $u \in \mathbb{R}^{n}$ respectively. When used in conjunction with matrix notation, $\mathrm{D}$ and $\mathrm{D}_{u}$ represent a row operation:

$$
\mathrm{D}=\left[\partial / \partial U^{1}, \ldots, \partial / \partial U^{N}\right], \quad \mathrm{D}_{u}=\left(\partial / \partial u^{1}, \ldots, \partial / \partial u^{n}\right) .
$$

(3) The symbol $\partial_{\alpha}$ denotes the derivative with respect to $x_{\alpha}, \alpha=1, \ldots, d$. The summation convention over repeated indices is employed throughout the article: repeated indices are summed over the range $1, \ldots, d$.

Motivated by theoretical studies $[15,18,23]$ as well as computations devoted to the approximation of the hyperbolic systems of conservation laws and kinetic equations [5] by relaxation schemes, our analysis is based on the following assumptions:

- The manifold $\mathcal{M}$ of Maxwellians (the equilibrium solutions $U_{e q}$ to the equation $R(U)=0$ ) can be parametrized by $n$ conserved quantities

$$
U_{e q}=M(u), \quad u \in \mathbb{R}^{n} .
$$

- $\nabla R(U)$ satisfies the nondegeneracy condition

$$
\begin{cases}\operatorname{dim} & \mathcal{N}(\nabla R(M(u)))=n, \\ \operatorname{dim} & \mathcal{R}(\nabla R(M(u))) N-n .\end{cases}
$$

- There exists a projection matrix

$$
\mathbb{P}: \mathbb{R}^{N} \rightarrow \mathbb{R}^{n} \quad \text { with } \quad \operatorname{rank} \mathbb{P}=n
$$

corresponding to Maxwellians that determines the conserved quantity

$$
\begin{gathered}
u=\mathbb{P} U \text { and satisfies } \\
\mathbb{P} M(u)=u \text { and } \quad \mathbb{P} R(U)=0 \text { for all } u \in \mathbb{R}^{n}, U \in \mathbb{R}^{N} .
\end{gathered}
$$


In this case, the corresponding system of balance laws for conserved quantities is given by

$$
\partial_{t} u+\partial_{\alpha} \mathbb{P} F_{\alpha}(M(u))=\mathbb{P} G(M(u))
$$

which can be rewritten in the form

$$
\partial_{t} u+\partial_{\alpha} f_{\alpha}(u)=g(u)
$$

with $f$ and $g$ defined by

$$
f_{\alpha}(u):=\mathbb{P} F_{\alpha}(M(u)), \quad g(u):=\mathbb{P} G(M(u)) .
$$

The system of balance laws (2.1) results from applying $\mathbb{P}$ to $(1.2)$, letting $\varepsilon \rightarrow 0$, and then using the fact that at the equilibrium $U_{e q}=M(u), u=\mathbb{P} U_{e q}$.

Our analysis exploits the entropy structure of the relaxation systems under consideration. Below are stated the main structural assumptions on (1.2).

2.1. Entropy structure. Some additional assumptions on the system (1.2) read:

- The system (1.2) is equipped with a globally defined entropy $H(U)$ and corresponding fluxes $Q_{\alpha}(U), \alpha=1, \ldots, d$, such that

$$
\begin{aligned}
& H: \mathbb{R}^{N} \rightarrow \mathbb{R} \text { is convex, } \\
& \mathrm{D} H(U) \mathrm{D} F_{\alpha}(U)=\mathrm{D} Q_{\alpha}(U) .
\end{aligned}
$$

- The entropy $H(U)$ is such that

$$
D(U):=-\mathrm{D} H(U) R(U) \geqslant 0, \quad U \in \mathbb{R}^{N} .
$$

The entropy equation for the relaxation system (1.2) in that case is given by

$$
\partial_{t} H(U)+\partial_{\alpha} Q_{\alpha}(U)=-\frac{1}{\varepsilon} \mathrm{D}(U)+\mathrm{D} H(U) G(U)
$$

- Entropy consistency. The restriction of the entropy pair $H, Q_{\alpha}$,

$$
\eta(u):=H(M(u)), \quad q_{\alpha}(u):=Q_{\alpha}(M(u)),
$$

on the equilibrium manifold $\mathcal{M}$ is an entropy pair $\eta-q_{\alpha}$ for the system of conserved quantities (2.1), that is,

$$
\mathrm{D}_{u} \eta(u) \mathrm{D}_{u} f_{\alpha}(u)=\mathrm{D}_{u} q_{\alpha}(u), \quad u \in \mathbb{R}^{n}
$$

In that case smooth solutions to (2.1) satisfy the additional balance law

$$
\partial_{t} H(M(u))+\partial_{\alpha} Q_{\alpha}(M(u))=\mathrm{D}_{u} \eta(u) g(u)
$$

In the sequel, we present some implications on the geometry of the manifold $\mathcal{M}$ obtained as a consequence of the entropy structure of the relaxation systems. We refer the reader to [23] for the details of the derivation in a relevant setting. 
2.2. Properties of $H$ and $Q_{\alpha}$ on the manifold $\mathcal{M}$. The geometric implications of the assumptions

$$
\begin{aligned}
& \mathrm{D} H(U) R(U) \leqslant 0, \quad R(M(u))=0, \\
& \operatorname{rank} \mathbb{P}=n, \quad \mathbb{P}(M(u))=u, \quad \forall u \in \mathbb{R}^{n}, U \in \mathbb{R}^{N}
\end{aligned}
$$

are the following [23]:

$$
\begin{aligned}
\mathcal{R}(\mathrm{D} R(M(u))) & =\mathcal{N}(\mathbb{P}), \\
\mathrm{D} H(M(u))[\mathrm{D} R(M(u)) A] & =0, \quad \forall u \in \mathbb{R}^{n}, A \in R^{N}, \\
\mathrm{D} H(M(u)) V & =0, \quad \forall V \in R^{N} \text { with } \mathbb{P} V=0 .
\end{aligned}
$$

Thus, the entropy consistency hypothesis (H6) along with the property $(2.5)_{3}$ imply that the gradients of entropies $\eta, H$ are related by

$$
\mathrm{D}_{u} \eta(u) \mathbb{P} A=\mathrm{D} H(M(u)) A, \quad \forall A \in \mathbb{R}^{N} .
$$

Then, in view of $(2.2)_{2}$, we have

$$
\mathrm{D}_{u} \eta(u) g(u)=\mathrm{D} H(M(u)) G(M(u)), \quad \forall u \in \mathbb{R}^{n},
$$

and thus the entropy equation (2.4) for conserved quantities may be written as

$$
\partial_{t} H(M(u))+\partial_{\alpha} Q_{\alpha}(M(u))=\mathrm{D} H(M(u)) G(M(u)) .
$$

2.3. Dissipation. Making use of the dissipation incorporated in the term $D(U)=-D H(U) R(U)$ we introduce an additional hypothesis which plays the role of relative dissipation, a measure of the distance between a relaxation state vector $U \in \mathbb{R}^{N}$ and its "equilibrium version" $M(u) \in \mathbb{R}^{N}$ with $u=\mathbb{P}(U)$ on the manifold of Maxwellians $\mathcal{M}$. More precisely,

- We assume that for some $\nu>0$

$$
-[\mathrm{D} H(U)-\mathrm{D} H(M(u))][R(U)-R(M(u))] \geqslant \nu|U-M(u)|^{2}
$$

for arbitrary $U \in \mathbb{R}^{N}$ with $u=\mathbb{P} U$.

Note that (H7) is stronger then the following assumption:

- For every ball $B_{r} \subset \mathbb{R}^{N}$ there exists $\nu_{r}>0$ such that

$$
-[\mathrm{D} H(U)-\mathrm{D} H(M(u))][R(U)-R(M(u))] \geqslant \nu_{r}|U-M(u)|^{2}
$$

for $U, M(u) \in B_{r}$, where $u=\mathbb{P} U$,

which will be of use in Theorem 3.3.

Our analysis handles a large class of source terms. The following hypothesis will be relevant to our subsequent discussion.

- The source term $G(U)$ is weakly dissipative with respect to the manifold $\mathcal{M}$ in the sense of Definition 2.1.

Definition 2.1. We say that the source $G(U)$ is weakly dissipative with respect to the manifold $\mathcal{M}$, if for all arbitrary $U, M(\bar{u}) \in \mathbb{R}^{N}$

$$
-[\mathrm{D} H(U)-\mathrm{D} H(M(\bar{u}))][G(U)-G(M(\bar{u}))] \geqslant 0 .
$$

An alternative condition on the source $G(U)$, exploited in Theorem 3.2, reads:

- For every compact set $\mathcal{A} \subset \mathbb{R}^{N}$ there exists $L_{\mathcal{A}}>0$ such that

$$
|G(U)-G(\bar{U})| \leqslant L_{\mathcal{A}}|U-\bar{U}| \text { for all } U \in \mathbb{R}^{N}, \bar{U} \in \mathcal{A} \text {. }
$$


2.4. Source consistency. We first note that the hypothesis (H8) is less restrictive than the requirement for $G$ to be weakly dissipative, hence a special name for it: $\mathcal{M}$-weakly dissipativity.

We next point out that $(\mathrm{H} 8)$ requires a certain consistency between the source terms $G(U)$ and $g(u)$ which are related by (2.2). Namely, take an arbitrary $u \in \mathbb{R}^{n}$ and set $U=M(u)$ in (H8). Then, recalling (2.6) one concludes that (H8) implies that the source $g(u)$ in the system (2.1) is weakly dissipative, that is

$$
-\left(\mathrm{D}_{u} \eta(u)-\mathrm{D}_{u} \eta(\bar{u})\right)(g(u)-g(\bar{u})) \geqslant 0, \quad u, \bar{u} \in \mathbb{R}^{n} .
$$

Thus, (H8) makes sense only when the source $g(u)$ in the equilibrium system (2.1) is weakly dissipative in the sense of (2.8).

2.5. Weak solutions and entropy admissibility. We introduce the notions of weak solutions and entropy admissibility following the discussion in [12, Section 4.3, 4.5].

Definition 2.2. A locally bounded measurable function $U(x, t)$, defined on $\mathbb{R}^{d} \times[0, T)$ and taking values in an open set $\mathcal{O} \subset \mathbb{R}^{N}$, is a weak solution to

$$
\partial_{t} U+\partial_{\alpha} F_{\alpha}(U)=\frac{1}{\varepsilon} R(U)+G(U), \quad U(x, 0)=U_{0}(x),
$$

with $F, R, G: \mathcal{O} \rightarrow \mathbb{R}^{N}$ Lipschitz, if

$$
\begin{aligned}
\int_{0}^{T} \int_{\mathbb{R}^{d}}\left\{\partial_{t} \widehat{\Phi} U+\partial_{\alpha} \widehat{\Phi} F_{\alpha}(U)\right\} d x d t+\int_{\mathbb{R}^{d}} \widehat{\Phi}(x, 0) U_{0}(x) d x \\
+\int_{0}^{T} \int_{\mathbb{R}^{d}} \widehat{\Phi}(x, t)\left[\frac{1}{\varepsilon} R(U)+G(U)\right] d x d t=0
\end{aligned}
$$

for every Lipschitz test function $\widehat{\Phi}(x, t)$ with compact support in $\mathbb{R}^{d} \times[0, T)$ and values in $\mathbb{M}^{1 \times N}$.

Definition 2.3. Assume that $H, Q_{\alpha}$ is an entropy-entropy flux pair of (2.9). Then a weak solution $U(x, t)$ of (2.9), in the sense of Definition 2.2, defined in $\mathbb{R}^{d} \times[0, T)$, is entropy admissible relative to $H$ if

$$
\begin{aligned}
\int_{0}^{T} \int_{\mathbb{R}^{d}}\left\{\partial_{t} \varphi H(U)+\right. & \left.\partial_{\alpha} \varphi Q_{\alpha}(U)\right\} d x d t+\int_{\mathbb{R}^{d}} \varphi(x, 0) H\left(U_{0}(x)\right) d x \\
& +\int_{0}^{T} \int_{\mathbb{R}^{d}} \varphi(x, t) \mathrm{D} H(U)\left[\frac{1}{\varepsilon} R(U)+G(U)\right] d x d t \geqslant 0
\end{aligned}
$$

for every nonnegative Lipschitz test function $\varphi(x, t)$ with compact support in $\mathbb{R}^{d} \times$ $[0, T)$.

REMARK 2.1. Note, a smooth solution $U^{\varepsilon}$ of (1.2) satisfies (2.11) identically as an equality and therefore is admissible. It is worth pointing out that relaxation systems of type (1.2) are often designed to produce global smooth solutions. We refer the reader to $[16,26]$ as well as $[12$, Section 5.2] for further remarks. A more detailed discussion about the existence of smooth solutions follows in the sequel.

\section{Main results}

In this section we present the main results of this article. 


\section{1. $\mathcal{M}$-weakly dissipative source $G(U)$.}

THEOREM 3.1. Let $\bar{u}(x, t)$ be a smooth solution of the equilibrium system (2.1), defined on $\mathbb{R}^{d} \times[0, T]$, with initial data $\bar{u}_{0}(x)$. Let $\left\{U^{\varepsilon}(x, t)\right\}$ be a family of admissible weak solutions of the relaxation system (1.2) on $\mathbb{R}^{d} \times[0, T)$, with initial data $U_{0}^{\varepsilon}(x)$, and let $u^{\varepsilon}(x, t)=\mathbb{P} U^{\varepsilon}(x, t)$ denote the conserved quantity associated to $U^{\varepsilon}$.

Assume (H1)-(H8) hold and suppose that:

(i) $H(U), F(U)$ in the relaxation system (1.2) satisfy for some $M, \mu, \mu^{\prime}>0$

$$
\mu \mathbf{I} \leqslant \mathrm{D}^{2} H(U) \leqslant \mu^{\prime} \mathbf{I}, \quad\left|\mathrm{D} F_{\alpha}(U)\right|<M, \quad U \in \mathbb{R}^{N} .
$$

(ii) $\eta(u)=H(M(u)), f(u)=\mathbb{P} F(M(u))$ satisfy for some $K>0$

$$
\left|\mathrm{D}_{u}^{3} \eta(u)\right| \leqslant K, \quad\left|\mathrm{D}_{u}^{2} f(u)\right| \leqslant K, \quad u \in \mathbb{R}^{n} .
$$

Then, for $R>0$ there exist constants $C=C(R, T, \nabla \bar{u}, M, K)>0$ and $s>0$ independent of $\varepsilon$ such that

$$
\int_{|x|<R} H^{r}(x, t) d x \leqslant C\left(\int_{|x|<R+s t} H^{r}(x, 0) d x+\varepsilon\right), \quad \text { a.e. } t \in[0, T) .
$$

Moreover, if the initial data satisfy

$$
\int_{|x|<R+s T} H^{r}(x, 0) d x \rightarrow 0 \quad \text { as } \varepsilon \downarrow 0,
$$

then

$$
\underset{t \in[0, T)}{\operatorname{esssup}} \int_{|x|<R}\left|U^{\varepsilon}-M(\bar{u})\right|^{2}(x, t) d x \rightarrow 0 \quad \text { as } \varepsilon \downarrow 0 .
$$

3.2. General source $G(U)$. We now drop the assumption (H8) which leads to the following theorem.

TheOrem 3.2. Let $\bar{u}$ be a smooth solution of $(2.1)$, defined on $\mathbb{R}^{d} \times[0, T]$, with initial data $\bar{u}_{0}$, and $\left\{U^{\varepsilon}\right\}$ a family of admissible weak solutions of (1.2) on $\mathbb{R}^{d} \times[0, T)$, with initial data $U_{0}^{\varepsilon}$.

Assume (H1)-(H7), (H9) hold. Suppose that $H(U), F(U), \eta(u)$, and $f(u)$ satisfy (i)-(ii) of Theorem 3.1. Then, for $R>0$ there holds the estimate (ER) for some constants $C=C(R, T, \nabla \bar{u}, M, K, L)>0$ and $s=s\left(M, \mu^{\prime}\right)>0$ independent of $\varepsilon$. Moreover, if the initial data satisfy $(\mathrm{CD})$, then (CS) holds.

3.3. Uniformly bounded $\bar{u},\left\{U^{\varepsilon}\right\}$. If a priori bounds on the family of solutions $\left\{U^{\varepsilon}\right\}$ are available, then it is possible to weaken the requirements $(i)-(i i)$ of theorems 3.1, 3.2. For example, one may weaken the assumption for $H$ to be uniformly convex and $\mathrm{D} F_{\alpha}, \mathrm{D}_{u}^{2} f_{\alpha}$, and $\mathrm{D}_{u}^{3} \eta$ to be uniformly bounded.

THEOREM 3.3. Let $\bar{u}$ be a smooth solution of (2.1), defined on $\mathbb{R}^{d} \times[0, T]$, with initial data $\bar{u}_{0}$, and $\left\{U^{\varepsilon}\right\}$ a family of admissible weak solutions of $(1.2)$ on $\mathbb{R}^{d} \times[0, T)$, with initial data $U_{0}^{\varepsilon}$.

Assume (H1)-(H6), (H7*) hold. Suppose that: 
(i) $\left\{U^{\varepsilon}\right\},\left\{M\left(u^{\varepsilon}\right)\right\}$, and $M(\bar{u})$ take values in a ball $B_{r} \subset \mathbb{R}^{N}$.

(ii) $H(U) \in C^{2}\left(\mathbb{R}^{N}\right)$ is strictly convex. $F(U), \eta(u), f(u)$ are smooth.

(iii) The source $G(U)$ either satisfies (H8) or is locally Lipschitz.

Then, for $R>0$ the estimate (ER) holds for some constants

$$
C=C\left(R, T, B_{r},\|\nabla \bar{u}\|_{W^{1, \infty}\left(\mathcal{C}_{(T, R)}\right)}\right)>0
$$

and

$$
s=\mu_{r}{ }^{-1} \sup _{U, V \in B_{r}} \sum_{\alpha}\left|\mathrm{D}^{2} H(U) \mathrm{D} F_{\alpha}(V)\right|,
$$

where $\mathcal{C}_{(T, R)}$ denotes a cone

$$
\mathcal{C}_{(T, R)}=\{(x, t): 0<t<T,|x|<R+s(T-t)\},
$$

and $\mu_{r}>0$ is a constant such that

$$
\mu_{r} \mathbf{I}<\mathrm{D}^{2} H(U), \quad U \in B_{r} .
$$

Moreover, if the initial data satisfy (CD), then (CS) holds.

\section{Relative entropy}

To compare the solution $U^{\varepsilon}$ of the relaxation system (1.2) and the solution $\bar{u}$ of the equilibrium system (2.1), we employ the notion of the relative entropy [9]. We define the relative entropy and entropy-fluxes [23] among the two solutions by

$$
\begin{aligned}
& H^{r}\left(U^{\varepsilon}, M(\bar{u})\right):=H\left(U^{\varepsilon}\right)-H(M(\bar{u}))-\mathrm{D} H(M(\bar{u}))\left[U^{\varepsilon}-M(\bar{u})\right], \\
& Q_{\alpha}^{r}\left(U^{\varepsilon}, M(\bar{u})\right):=Q_{\alpha}\left(U^{\varepsilon}\right)-Q_{\alpha}(M(\bar{u}))-\mathrm{D} H(M(\bar{u}))\left[F_{\alpha}\left(U^{\varepsilon}\right)-F_{\alpha}(M(\bar{u}))\right] .
\end{aligned}
$$

By (H5) we have

$$
D\left(U^{\varepsilon}\right)=-\mathrm{D} H\left(U^{\varepsilon}\right) R\left(U^{\varepsilon}\right) \geqslant 0,
$$

which expresses the entropy dissipation of the relaxation system (1.2). In view of $(2.5)_{3}$ and the fact that $R(M(u))=0$ for all $u \in \mathbb{R}^{n}, D\left(U^{\varepsilon}\right)$ may be written in an alternative form

$$
D\left(U^{\varepsilon}\right)=-\left[\mathrm{D} H\left(U^{\varepsilon}\right)-\mathrm{D} H\left(M\left(u^{\varepsilon}\right)\right)\right]\left[R\left(U^{\varepsilon}\right)-R\left(M\left(u^{\varepsilon}\right)\right)\right] \geqslant 0,
$$

where $u^{\varepsilon}=\mathbb{P} U^{\varepsilon}$. Finally, we denote by

$$
S\left(U^{\varepsilon}, M(\bar{u})\right):=-\left[\mathrm{D} H\left(U^{\varepsilon}\right)-\mathrm{D} H(M(\bar{u}))\right]\left[G\left(U^{\varepsilon}\right)-G(M(\bar{u}))\right]
$$

the term (not necessarily dissipative) associated with the source $G(U)$.

Let $U \equiv U^{\varepsilon}(x, t)$ be a smooth solution of the relaxation system $(1.2), u(x, t)=$ $\mathbb{P} U(x, t)$ be the conserved quantity associated to $U$, and $\bar{u}(x, t)$ be a smooth solution of the equilibrium system (2.1). Then the relative entropy $H^{r}(U, M(\bar{u}))$ satisfies the following.

LEMMA 4.1 (Relative entropy identity). Suppose $\bar{u}(x, t)$ is a smooth solution of the equilibrium system (2.1), defined on $\mathbb{R}^{d} \times[0, T]$, with initial data $\bar{u}_{0}(x)$. Let $U \equiv U^{\varepsilon}(x, t)$ be any admissible weak solution of the relaxation system (1.2) on $\mathbb{R}^{d} \times$ 
$[0, T)$, with initial data $U_{0}(x)$, and let $u(x, t)=\mathbb{P} U(x, t)$ denote the conserved quantity associated to $U$. Then the relative entropy $H^{r}(U, M(\bar{u}))$ satisfies

$$
\begin{aligned}
& \int_{0}^{T} \int_{\mathbb{R}^{d}}\left\{-\partial_{t} \varphi H^{r}(U, \bar{u})-\partial_{\alpha} \varphi Q_{\alpha}^{r}(U, \bar{u})\right\} d x d t-\int_{\mathbb{R}^{d}} \varphi(x, 0) H^{r}\left(U_{0}, M\left(\bar{u}_{0}\right)\right) d x \\
\leqslant & \int_{0}^{T} \int_{\mathbb{R}^{d}} \varphi\left\{-\frac{1}{\varepsilon} D-S+J_{1}+J_{2}+J_{3}+J_{4}\right\} d x d t
\end{aligned}
$$

for every nonnegative Lipschitz test function $\varphi(x, t)$ with compact support in $\mathbb{R}^{d} \times$ $[0, T)$, where

$$
\begin{aligned}
& J_{1}:=-\left(\mathrm{D}_{u}^{2} \eta(\bar{u}) \partial_{\alpha} \bar{u}\right)^{\top}\left(f_{\alpha}(u)-f_{\alpha}(\bar{u})-\mathrm{D}_{u} f_{\alpha}(\bar{u})(u-\bar{u})\right), \\
& J_{2}:=-\left(\mathrm{D}_{u}^{2} \eta(\bar{u}) \partial_{\alpha} \bar{u}\right)^{\top} \mathbb{P}\left[F_{\alpha}(U)-F_{\alpha}(M(u))\right], \\
& J_{3}:=g(\bar{u})^{\top}\left(\mathrm{D}_{u} \eta(u)^{\top}-\mathrm{D}_{u} \eta(\bar{u})^{\top}-\mathrm{D}_{u}^{2} \eta(\bar{u})^{\top}(u-\bar{u})\right), \\
& J_{4}:=[\mathrm{D} H(U)-\mathrm{D} H(M(u))] G(M(\bar{u})) .
\end{aligned}
$$

If, in addition, $\left\{U^{\varepsilon}\right\}$ are smooth solutions, then they identically satisfy (2.11) as equality. As a consequence, the inequality (4.5) for the relative entropy $H^{r}$ becomes the identity

$$
\partial_{t} H^{r}+\partial_{\alpha} Q_{\alpha}^{r}+\frac{1}{\varepsilon} D+S=J_{1}+J_{2}+J_{3}+J_{4}, \quad(x, t) \in \mathbb{R}^{d} \times[0, T) .
$$

Proof. Let us fix any nonnegative, Lipschitz continuous test function $\varphi(x, t)$, compactly supported in $\mathbb{R}^{d} \times[0, T)$. Because $\bar{u}$ is smooth, from (2.7) it follows that $\eta(\bar{u})=H(M(\bar{u}))$ satisfies the entropy identity

$$
\partial_{t} H(M(\bar{u}))+\partial_{\alpha} Q_{\alpha}(M(\bar{u}))=\mathrm{D} H(M(\bar{u})) G(M(\bar{u})),
$$

which in its the weak form reads

$$
\begin{aligned}
& \int_{0}^{T} \int_{\mathbb{R}^{d}}\left(\partial_{t} \varphi H(M(\bar{u}))+\partial_{\alpha} \varphi Q_{\alpha}(M(\bar{u}))\right) d x d t \\
& +\int_{\mathbb{R}^{d}} \varphi(x, 0) H\left(M\left(\bar{u}_{0}\right)\right) d x+\int_{0}^{T} \int_{\mathbb{R}^{d}} \varphi \mathrm{D} H(M(\bar{u})) G(M(\bar{u})) d x d t=0 .
\end{aligned}
$$

Recall that $U$, an admissible weak solution of (1.2) with initial data $U_{0}$, must satisfy the inequality (2.11). Thus, upon subtracting (4.8) from (2.11), we obtain

$$
\begin{aligned}
& \int_{0}^{T} \int_{\mathbb{R}^{d}}\left\{\partial_{t} \varphi(H(U)-H(M(\bar{u})))\right.\left.+\partial_{\alpha} \varphi\left(Q_{\alpha}(U)-Q_{\alpha}(M(\bar{u}))\right)\right\} d x d t \\
&+\int_{0}^{T} \int_{\mathbb{R}^{d}} \varphi\left\{\mathrm{D} H(U)\left[\frac{1}{\varepsilon} R(U)+G(U)\right]-\mathrm{D} H(M(\bar{u})) G(M(\bar{u}))\right\} d x d t \\
&+\int_{\mathbb{R}^{d}} \varphi(x, 0)\left(H\left(U_{0}\right)-H\left(M\left(\bar{u}_{0}\right)\right)\right) d x \geqslant 0 .
\end{aligned}
$$

Next, recalling that $\bar{u}$ is a smooth solution of

$$
\partial_{t} \bar{u}+\partial_{\alpha} \mathbb{P} F_{\alpha}(M(\bar{u}))=\mathbb{P} G(M(\bar{u}))
$$


and that $\mathbb{P} M(\bar{u})=\bar{u}$, we obtain the identity

$$
\begin{aligned}
\int_{0}^{T} \int_{\mathbb{R}^{d}}\left\{\partial_{t} \Phi \mathbb{P} M(\bar{u})+\partial_{\alpha} \Phi \mathbb{P} F_{\alpha}(M(\bar{u}))\right\} d x d t \\
\quad+\int_{\mathbb{R}^{d}} \Phi(x, 0) \mathbb{P} M\left(\bar{u}_{0}(x)\right) d x+\int_{0}^{T} \int_{\mathbb{R}^{d}} \Phi(x, t) \mathbb{P} G(M(\bar{u})) d x d t=0,
\end{aligned}
$$

where $\Phi(x, t)$ is a Lipschitz continuous vector field with compact support in $\mathbb{R}^{d} \times[0, T)$ and values in $\mathbb{M}^{1 \times n}$. Also, because $U$ is a weak solution of (1.2), it must satisfy (2.10) which, with $\widehat{\Phi}=\Phi \mathbb{P} \in \mathbb{M}^{1 \times N}$, reads

$$
\begin{aligned}
\int_{0}^{T} \int_{\mathbb{R}^{d}} & \left\{\partial_{t} \Phi \mathbb{P} U+\partial_{\alpha} \Phi \mathbb{P} F_{\alpha}(U)\right\} d x d t \\
& +\int_{\mathbb{R}^{d}} \Phi(x, 0) \mathbb{P} U_{0}(x) d x+\int_{0}^{T} \int_{\mathbb{R}^{d}} \Phi(x, t) \mathbb{P} G(U) d x d t=0
\end{aligned}
$$

in view of the property $\mathbb{P} R(U)=0$.

Now, we subtract (4.12) from (4.11), set the Lipschitz continuous vector field $\Phi=\varphi \mathrm{D}_{u} \eta(\bar{u})$, and recall the geometric relation (2.6), to get

$$
\begin{gathered}
\int_{0}^{T} \int_{\mathbb{R}^{d}}\left\{\partial_{t} \varphi \mathrm{D} H(M(\bar{u}))[U-M(\bar{u})]\right. \\
\left.+\partial_{\alpha} \varphi \mathrm{D} H(M(\bar{u}))\left[F_{\alpha}(U)-F_{\alpha}(M(\bar{u}))\right]\right\} d x d t \\
+\int_{0}^{T} \int_{\mathbb{R}^{d}} \varphi\left\{\left(\mathrm{D}_{u}^{2} \eta(\bar{u}) \partial_{t} \bar{u}\right)^{\top} \mathbb{P}[U-M(\bar{u})]\right. \\
+\left(\mathrm{D}_{u}^{2} \eta(\bar{u}) \partial_{\alpha} \bar{u}\right)^{\top} \mathbb{P}\left[F_{\alpha}(U)-F_{\alpha}(M(\bar{u}))\right] \\
+\mathrm{D} H(M(\bar{u}))[G(U)-G(M(\bar{u}))]\} d x d t \\
+\int_{\mathbb{R}^{d}} \varphi(x, 0) \mathrm{D} H\left(M\left(\bar{u}_{0}\right)\right)\left[U_{0}(x)-M\left(\bar{u}_{0}\right)\right] d x=0 .
\end{gathered}
$$

The existence of an entropy pair $\eta-q_{\alpha}$ is equivalent to the property

$$
\mathrm{D}_{u}^{2} \eta(v) \mathrm{D}_{u} f_{\alpha}(v)=\mathrm{D}_{u} f_{\alpha}(v)^{\top} \mathrm{D}_{u}^{2} \eta(v), \quad \forall v \in \mathbb{R}^{n}
$$

and therefore, in view of (4.10), we have

$$
\begin{aligned}
\mathrm{D}_{u}^{2} \eta(\bar{u}) \partial_{t} \bar{u} & =\mathrm{D}_{u}^{2} \eta(\bar{u})\left(-\mathrm{D}_{u} f_{\alpha}(\bar{u}) \partial_{\alpha} \bar{u}+g(\bar{u})\right) \\
& =-\mathrm{D}_{u} f_{\alpha}(\bar{u})^{\top} \mathrm{D}_{u}^{2} \eta(\bar{u}) \partial_{\alpha} \bar{u}+\mathrm{D}_{u}^{2} \eta(\bar{u}) g(\bar{u}) .
\end{aligned}
$$

Hence we must have

$$
\begin{gathered}
\left(\mathrm{D}_{u}^{2} \eta(\bar{u}) \partial_{t} \bar{u}\right)^{\top} \mathbb{P}[U-M(\bar{u})]+\left(\mathrm{D}_{u}^{2} \eta(\bar{u}) \partial_{\alpha} \bar{u}\right)^{\top} \mathbb{P}\left[F_{\alpha}(U)-F_{\alpha}(M(\bar{u}))\right] \\
=\left(\mathrm{D}_{u}^{2} \eta(\bar{u}) \partial_{\alpha} \bar{u}\right)^{\top}\left(f_{\alpha}(u)-f_{\alpha}(\bar{u})-\mathrm{D}_{u} f_{\alpha}(\bar{u})(u-\bar{u})\right) \\
\quad+\left(\mathrm{D}_{u}^{2} \eta(\bar{u}) \partial_{\alpha} \bar{u}\right)^{\top} \mathbb{P}\left[F_{\alpha}(U)-F_{\alpha}(M(u))\right]+\left(\mathrm{D}_{u}^{2} \eta(\bar{u}) g(\bar{u})\right)^{\top}(u-\bar{u}),
\end{gathered}
$$

where we used (2.2), the fact that $u=\mathbb{P} U$, and $\mathbb{P} M(\bar{u})=\bar{u}$. 
Combining (4.9) with (4.13)-(4.14) and recalling (4.2), (4.3) we obtain

$$
\begin{array}{r}
\int_{0}^{T} \int_{\mathbb{R}^{d}}\left\{\partial_{t} \varphi H^{r}(U, \bar{u})+\partial_{\alpha} \varphi Q_{\alpha}^{r}(U, \bar{u})\right\} d x d t \\
+\int_{0}^{T} \int_{\mathbb{R}^{d}} \varphi\left\{-\frac{1}{\varepsilon} D-S+J_{1}+J_{2}+J_{4}-\left(\mathrm{D}_{u}^{2} \eta(\bar{u}) g(\bar{u})\right)^{\top}(u-\bar{u})\right. \\
+[\mathrm{D} H(M(u))-\mathrm{D} H(M(\bar{u}))] G(M(\bar{u}))\} d x d t \\
+\int_{\mathbb{R}^{d}} \varphi(x, 0) H^{r}\left(U_{0}, M\left(\bar{u}_{0}\right)\right) d x \geqslant 0 .
\end{array}
$$

Observe that, in view of $(2.2),(2.6)$, we have

$$
[\mathrm{D} H(M(u))-\mathrm{D} H(M(\bar{u}))] G(M(\bar{u}))=\left(\mathrm{D}_{u} \eta(u)-\mathrm{D}_{u} \eta(\bar{u})\right) g(\bar{u}),
$$

and hence

$$
\begin{aligned}
& {[\mathrm{D} H(M(u))-\mathrm{D} H(M(\bar{u}))] G(M(\bar{u}))-\left(\mathrm{D}_{u}^{2} \eta(\bar{u}) g(\bar{u})\right)^{\top}(u-\bar{u}) } \\
= & g(\bar{u})^{\top}\left(D_{u} \eta(u)^{\top}-\mathrm{D}_{u} \eta(\bar{u})^{\top}-\mathrm{D}_{u}^{2} \eta(\bar{u})^{\top}(u-\bar{u})\right)=J_{3} .
\end{aligned}
$$

Then from (4.15) and (4.16) we get the desired inequality (4.5).

REMARK 4.1. Note that if $\left\{U^{\varepsilon}\right\}$ in Lemma 4.1 are smooth solutions of (2.1), then they identically satisfy (2.11) as equality and hence are admissible. As a consequence, the inequality (4.5) for the relative entropy $H^{r}$ becomes the identity

$$
\partial_{t} H^{r}+\partial_{\alpha} Q_{\alpha}^{r}+\frac{1}{\varepsilon} D+S=J_{1}+J_{2}+J_{3}+J_{4}, \quad(x, t) \in \mathbb{R}^{d} \times[0, T) .
$$

\section{Proof of theorems via error estimates}

To investigate the convergence of solutions $\left\{U^{\varepsilon}\right\}$ of the relaxation system (1.2) to $M(\bar{u})$ in the smooth regime, one employs the inequality (4.5) derived in the previous section. The preliminary analysis of the inequality indicates that the evolution of $H^{r}(\cdot, t)$ depends heavily on the properties of the entropy $H(U)$, flux $F(U)$, dissipative source $R(U)$, and, especially, the source $G(U)$.

\subsection{Proof of Theorem 3.1.}

Proof. The argument follows along the lines of [10, Theorem 5.2.1]. Fix $\varepsilon>0$. Because $U^{\varepsilon}$ is an admissible weak solution of (1.2) it must satisfy (2.11). Then [12, Lemma 1.3.3] implies that the map $t \rightarrow H\left(U^{\varepsilon}(\cdot, t)\right)$ is continuous on $[0, T) \backslash \mathcal{F}$ in $L^{\infty}(\mathcal{A})$ weak* , for any compact subset $\mathcal{A} \subset \mathbb{R}^{d}$, where $\mathcal{F}$ is at most countable.

We now fix $R>0$ and any point $t \in[0, T)$ of $L^{\infty}$ weak $^{*}$ continuity of $H\left(U^{\varepsilon}(\cdot, t)\right)$ and let $\mathcal{C}_{(t, R)}$ denote the cone

$$
\mathcal{C}_{(t, R)}=\{(x, \tau): 0<\tau<t,|x|<R+s(t-\tau)\}
$$

where $s$ is a constant selected later. To prove the statement of the theorem we need to monitor the evolution of the quantity

$$
\Psi(\tau)=\Psi(\tau ; t, R):=\int_{|x|<R+s(t-\tau)} H^{r}(x, \tau) d x, \quad 0 \leqslant \tau \leqslant t .
$$


Clearly $U^{\varepsilon}, \bar{u}$ satisfy the assumptions of Lemma 4.1 and hence there holds the relative entropy inequality

$$
\begin{aligned}
& \int_{0}^{T} \int_{\mathbb{R}^{d}}\left\{-H^{r}(x, \tau) \partial_{t} \varphi-Q_{\alpha}^{r}(x, \tau) \partial_{\alpha} \varphi+\frac{1}{\varepsilon} \varphi D\right\} d x d \tau-\int_{\mathbb{R}^{d}} H^{r}(x, 0) \varphi(x, 0) d x \\
\leqslant & \int_{0}^{T} \int_{\mathbb{R}^{d}} \varphi\left\{-S+J_{1}+J_{2}+J_{3}+J_{4}\right\} d x d \tau,
\end{aligned}
$$

where $D, S, J_{k}, k=1, \ldots, 4$ defined by (4.4) and $\varphi$ is nonnegative Lipschitz continuous function compactly supported in $\mathbb{R}^{d} \times[0, T)$.

Because the family $\left\{U^{\varepsilon}\right\}$ together with $\bar{u}$ are not necessarily uniformly bounded, to handle the flux term $Q^{r}$ we need to exploit the uniform convexity of the entropy $H(U)$. From (4.1) and the assumption $(i)$ it follows that there exists $c_{1}>0$ independent of $\varepsilon$ such that

$$
H^{r}\left(U^{\varepsilon}, M(\bar{u})\right) \geqslant c_{1}\left|U^{\varepsilon}-M(\bar{u})\right|^{2} .
$$

Now, by $(4.1)_{2}$ the relative entropy flux $Q_{\alpha}^{r}$ may be written as

$$
\begin{aligned}
Q_{\alpha}^{r}\left(U^{\varepsilon}, M(\bar{u})\right)= & \int_{0}^{1} \mathrm{D} Q_{\alpha}(\hat{U}(\beta))\left[U^{\varepsilon}-M(\bar{u})\right] d \beta \\
& -\int_{0}^{1} \mathrm{D} H(M(\bar{u}))\left[\mathrm{D} F_{\alpha}(\hat{U}(\beta))\left[U^{\varepsilon}-M(\bar{u})\right]\right] d \beta,
\end{aligned}
$$

where $\widehat{U}(\beta):=\beta U^{\varepsilon}+(1-\beta) M(\bar{u})$. Recalling (H4) we have

$$
\mathrm{D} Q_{\alpha}(\widehat{U})\left[U^{\varepsilon}-M(\bar{u})\right]=\mathrm{D} H(\widehat{U}) \mathrm{D} F_{\alpha}(\widehat{U})\left[U^{\varepsilon}-M(\bar{u})\right]
$$

and hence (5.3) becomes

$$
\begin{aligned}
Q_{\alpha}^{r} & =\int_{0}^{1}[\mathrm{D} H(\hat{U}(\beta))-\mathrm{D} H(M(\bar{u}))]\left[\mathrm{D} F_{\alpha}(\widehat{U}(\beta))\left[U^{\varepsilon}-M(\bar{u})\right]\right] d \beta \\
& =\left[U^{\varepsilon}-M(\bar{u})\right]^{\top}\left(\int_{0}^{1} \int_{0}^{1} \beta \mathrm{D}^{2} H(\widetilde{U}) \mathrm{D} F_{\alpha}(\widehat{U}) d \gamma d \beta\right)\left[U^{\varepsilon}-M(\bar{u})\right],
\end{aligned}
$$

where $\widetilde{U}(\beta, \gamma):=\beta \gamma U^{\varepsilon}+(1-\beta \gamma) M(\bar{u})$. Then, from (5.4) and $(i)$ we conclude that

$$
\sum_{\alpha}\left|Q_{\alpha}^{r}\right| \leqslant c_{2}\left|U^{\varepsilon}-M(\bar{u})\right|^{2}
$$

for some $c_{2}=c_{2}\left(M, \mu^{\prime}\right)>0$ independent of $\varepsilon$. Hence, in view of (5.2) and (5.5), we can choose $s>0$ such that

$$
s H^{r}(x, \tau)+\sum_{\alpha} \frac{x_{\alpha}}{|x|} Q_{\alpha}^{r}(x, \tau)>0, \quad(x, \tau) \in \mathbb{R}^{d} \times[0, T) .
$$

Next, take $\delta>0$ such that $t+\delta<T$ and select the test function $\varphi=\varphi(x, \tau)$ as follows (cf. [12, Theorem 5.3.1]):

$$
\varphi(x, \tau)=\theta(\tau) \gamma(x, \tau)
$$


where

$$
\begin{gathered}
\theta(\tau)=\left\{\begin{array}{lc}
1, & 0 \leqslant \tau<t, \\
1-\frac{1}{\delta}(\tau-t), & t \leqslant \tau \leqslant t+\delta, \\
0, & t+\delta \leqslant \tau,
\end{array}\right. \\
\gamma(x, \tau)= \begin{cases}1, & \tau>0,|x|-R-s(t-\tau)<0, \\
1-\frac{1}{\delta}(|x|-R-s(t-\tau)), & \tau>0,0<|x|-s(t-\tau)-R<\delta, \\
0, & \tau>0, \delta<|x|-R-s(t-\tau),\end{cases}
\end{gathered}
$$

and use it in (5.1). This gives

$$
\begin{aligned}
& \frac{1}{\delta} \int_{t}^{t+\delta} \int_{|x|<R} H^{r}(x, \tau) d x d \tau-\int_{|x|<R+s t} H^{r}(x, 0) d x \\
& \quad+\frac{1}{\delta} \int_{0}^{t} \int_{0<|x|-R-s(t-\tau)<\delta}\left(s H^{r}(x, \tau)+\sum_{\alpha} \frac{x_{\alpha}}{|x|} Q_{\alpha}^{r}(x, \tau)\right) d x d \tau \\
& \quad+\frac{1}{\varepsilon} \int_{0}^{t} \int_{|x|<R+s(t-\tau)} D d x d \tau+O(\delta) \\
& =\int_{0}^{t} \int_{|x|<R+s(t-\tau)}\left(-S+J_{1}+J_{2}+J_{3}+J_{4}\right) d x d \tau .
\end{aligned}
$$

We next let $\delta \rightarrow 0^{+}$in (5.7). The second integral in (5.7) is nonnegative in view of (5.6). Recalling $(\mathrm{H} 7)$ and using the fact that $H^{r}\left(U^{\varepsilon}(\cdot, \tau), \bar{u}(\cdot, \tau)\right)$ is weak* continuous in $L^{\infty}$ at $\tau=t$ we conclude

$$
\begin{aligned}
& \int_{|x|<R} H^{r}(x, t) d x+\frac{\nu}{\varepsilon} \iint_{\mathcal{C}_{(t, R)}}\left|U^{\varepsilon}-M\left(u^{\varepsilon}\right)\right|^{2} d x d \tau+\iint_{\mathcal{C}_{(t, R)}} S d x d \tau \\
\leqslant & \int_{|x|<R+s t} H^{r}(x, 0) d x+\iint_{\mathcal{C}_{(t, R)}}\left(\left|J_{1}\right|+\left|J_{2}\right|+\left|J_{3}\right|+\left|J_{4}\right|\right) d x d \tau .
\end{aligned}
$$

We next estimate the terms on the right-hand side of (5.8). Recalling (4.6) and using $(i),(i i)$, and Young's inequality we obtain

$$
\begin{aligned}
& \iint_{\mathcal{C}_{(t, R)}}\left|J_{1}\right|+\left|J_{3}\right| d x d \tau \leqslant C \iint_{\mathcal{C}_{(t, R)}}\left|U^{\varepsilon}-M(\bar{u})\right|^{2} d x d \tau, \\
& \iint_{\mathcal{C}_{(t, R)}}\left|J_{2}\right|+\left|J_{4}\right| d x d \tau \leqslant \frac{\nu}{\varepsilon} \iint_{\mathcal{C}_{(t, R)}}\left|U^{\varepsilon}-M(u)\right|^{2} d x+C \varepsilon,
\end{aligned}
$$

where the constant $C=C(t, R, u, \nabla \bar{u}, M, K)>0$ depends on the norms

$$
\|\bar{u}\|_{W^{1, \infty}\left(\mathcal{C}_{(t, R)}\right)}, \quad\|\bar{u}\|_{W^{1,2}\left(\mathcal{C}_{(t, R)}\right)}
$$

and constants $M$ and $K$ are introduced in $(i)$ and (ii). Finally, by (H8)

$$
\iint_{\mathcal{C}_{(t, R)}} S\left(U^{\varepsilon}, M(\bar{u})\right) d x d \tau \geqslant 0
$$


Then, combining (5.8)-(5.11) and recalling $(i)_{1}$ we conclude that

$$
\Psi(t ; t, R) \leqslant \Psi(0 ; t, R)+C\left(\varepsilon+\int_{0}^{t} \Psi(\tau ; t, R) d \tau\right) .
$$

Because $R>0$ and $t \in[0, T]$ in the above inequality are arbitrary, we conclude via the Gronwall lemma.

REMARK 5.1. The terms $J_{1}$ and $J_{3}$ (in the proof of Theorem 3.1) are bounded by $C H^{r}\left(U^{\varepsilon}, M(\bar{u})\right)$, in view of $(3.1)_{1}$ and $(5.9)_{1}$. This is one of the key features of the calculations that eventually leads to the use of the Gronwall lemma.

The term $S\left(U^{\varepsilon}, M(\bar{u})\right)$ has a "quadratic" structure similar to that of $J_{1}$ and $J_{3}$, and thus one may think that there is no need to require (H8). To this end, we point out that if (H8) does not hold, then one has to make sure that

$$
\iint_{\mathcal{C}_{(t, R)}} S\left(U^{\varepsilon}, M(\bar{u})\right) d x \leqslant c \iint_{\mathcal{C}_{(t, R)}} H^{r}\left(U^{\varepsilon}, M(\bar{u})\right) d x
$$

with $c=c(t, R)>0$ independent of $\varepsilon$ (in order to exploit the Gronwall lemma), and this is not true in general. In this case, to ensure (5.12), one has to impose certain regularity conditions on the source function $G(U)$.

5.2. Proof of Theorem 3.2. In this section we drop the assumption (H8) and following Remark 5.1 require the source $G(U)$ to satisfy (H9). This will ensure (5.12) and thus following the analysis in the proof of Theorem 3.1 we obtain the result.

5.3. Proof of Theorem 3.3. In the previous two sections we established convergence of weak solutions of the relaxation system (1.2) to the equilibrium system via the error estimate on the cone $\mathcal{C}_{(R, t)}$. Observe, however, that the bounds imposed on $\mathrm{D}^{2} H, \mathrm{D}_{u}^{3} \eta$ and $\mathrm{D}_{u} f_{\alpha}, \mathrm{D} F_{\alpha}$ in Theorem 3.1 are global. In particular, the requirement that $H$ is uniformly convex on $\mathbb{R}^{N}$ (which is used to handle the flux $Q_{\alpha}^{r}$ on the boundary of the cone; see (5.6)) is a very stringent condition that narrows significantly the class of systems to which our error analysis may be applied.

Let us note at this point that if a priori (local) bounds on the family of solutions $\left\{U^{\varepsilon}\right\}$ are available, then it is possible to weaken the requirements $(i)-(i i)$ of theorems 3.1 and 3.2. For example, one may weaken the assumption for $H$ to be uniformly convex and $\mathrm{D} F_{\alpha}, \mathrm{D}_{u}^{2} f_{\alpha}$, and $\mathrm{D}_{u}^{3} \eta$ to be uniformly bounded. This is indeed the case and the proof of Theorem 3.3 follows using the line of argument presented in the proof of Theorem 3.1.

\section{Application to elasticity}

Consider the relaxation of the (isothermal/isentropic) elasticity system:

$$
\left(\begin{array}{l}
u \\
v
\end{array}\right)_{t}-\left(\begin{array}{c}
v \\
\sigma(u)
\end{array}\right)_{x}=g(u, v)=\left(\begin{array}{c}
0 \\
g_{2}(u, v)
\end{array}\right)
$$

with the stress $\sigma(u)$ such that

$$
\sigma(0)=0 \quad \text { and } \quad 0<\gamma<\sigma^{\prime}(u)<\Gamma \text { for all } u \in \mathbb{R}^{n} .
$$

We assume that the source $g(u, v)$ satisfies one of the following: 
(i) Either $g$ is independent of $u$, that is $g(u, v)=g(v)$, and satisfies

$$
\left(g_{2}(v)-g_{2}(\bar{v})\right)(v-\bar{v}) \leqslant 0, \quad \forall v, \bar{v} \in \mathbb{R},
$$

(ii) or for every compact set $\mathcal{A} \subset \mathbb{R}^{2}$ there exists $L_{\mathcal{A}}>0$ such that

$$
\left|g_{2}(u, v)-g_{2}(\bar{u}, \bar{v})\right| \leqslant L_{\mathcal{A}}(|u-\bar{u}|+|v-\bar{v}|)
$$

for all $(u, v) \in \mathbb{R}^{2},(\bar{u}, \bar{v}) \in \mathcal{A}$.

The system (6.1) is equipped with the entropy-entropy flux pair $\bar{\eta}, \bar{q}$ given by

$$
\bar{\eta}(u, v)=\frac{1}{2} v^{2}+\Sigma(u), \quad \bar{q}(u, v)=-\sigma(u) v \quad \text { with } \quad \Sigma(u):=\int_{0}^{u} \sigma(\tau) d \tau .
$$

Relaxation via stress approximation. Consider the following extended system which approximates the stress $\sigma(u)$ :

$$
\left(\begin{array}{c}
u \\
v \\
\alpha
\end{array}\right)_{t}-\left(\begin{array}{c}
v \\
\alpha+E u \\
0
\end{array}\right)_{x}=\frac{1}{\varepsilon} R(u, v, \alpha)+G(u, v, \alpha)
$$

with

$$
R(u, v, \alpha)=(0,0, h(u)-\alpha)^{\top}, \quad G(u, v, \alpha)=\left(0, g_{2}(u, v), 0\right)^{\top},
$$

and the function $h(u)$ defined by

$$
h(u)=\sigma(u)-E u \quad \text { with } \quad E>\Gamma .
$$

Observe that as $\varepsilon \rightarrow 0$, the variable $\alpha$ tends to its equilibrium state $\alpha_{e q}=h(u)$. Thus, the corresponding equilibrium states $u_{e q}, v_{e q}$ satisfy (6.1). This motivates the parametrization of the manifold of Maxwellians by

$$
M(u, v, \alpha)=(u, v, h(u))^{\top},
$$

which implies (H1). Next, we easily check that

$$
\operatorname{dim} \mathcal{N}(\nabla R(M(u, v)))=2, \quad \operatorname{dim} \mathcal{R}(\nabla R(M(u, v)))=1
$$

which verifies (H2) for $n=2, N=3$. Also, the structure of (6.1) and (6.6) suggests the choice of the projection matrix

$$
\mathbb{P}=\left[\begin{array}{lll}
1 & 0 & 0 \\
0 & 1 & 0
\end{array}\right]
$$

for which $\mathbb{P} M(u, v)=(u, v)^{\top}, \mathbb{P} R(u, v, \alpha)=\mathbf{0}$, and hence (H3) is satisfied.

At this point, we identify the corresponding entropy-entropy flux pair of the system (6.6) and verify the remaining hypotheses that allow one to apply the theory developed in the preceding sections. In view of the requirement (6.2) for the stress $\sigma(u), h(u): \mathbb{R} \rightarrow \mathbb{R}$ is strictly decreasing, onto, and satisfies $h(0)=0$. Hence $h^{-1}: \mathbb{R} \rightarrow \mathbb{R}$ is well-defined. Then, we set

$$
\begin{aligned}
& H(u, v, \alpha):=\frac{1}{2} v^{2}+\frac{1}{2} E u^{2}+\alpha u-\int_{0}^{\alpha} h^{-1}(\xi) d \xi \\
& Q(u, v, \alpha):=-(\alpha+E u) v .
\end{aligned}
$$


It is easy to check that $H, Q$ is the entropy-entropy flux pair for the system (6.6). Next, we observe that the entropy $H$ maybe written as

$$
H(u, v, \alpha)=\frac{v^{2}}{2}+\frac{\gamma u^{2}}{4}+\psi(\alpha)+\frac{(\alpha+\widehat{E} u)^{2}}{2 \widehat{E}},
$$

where

$$
\psi(\alpha):=\int_{0}^{\alpha}\left(-h^{-1}(\xi)-\frac{\xi}{\widehat{E}}\right) d \xi, \quad \widehat{E}:=E-\frac{\gamma}{2}>0 .
$$

From (6.2) we have

$$
\begin{aligned}
\psi^{\prime \prime}(\alpha) & =-h^{-1^{\prime}}(\alpha)-\frac{1}{\widehat{E}} \\
& =\frac{1}{\left(E-\sigma^{\prime}\left(h^{-1}(\alpha)\right)\right)}-\frac{1}{\left(E-\frac{\gamma}{2}\right)} \geqslant \frac{\gamma}{2(E-\gamma)\left(E-\frac{\gamma}{2}\right)}>0 .
\end{aligned}
$$

Then (6.8) and (6.9) imply that there exist $\mu, \mu^{\prime}>0$ such that

$$
\mu \mathbf{I} \leqslant \mathrm{D}^{2} H(v, u, \alpha) \leqslant \mu^{\prime} \mathbf{I}, \quad(u, v, \alpha) \in \mathbb{R}^{3},
$$

and hence the pair $H, Q$ satisfies (H4). Next, we compute

$$
\mathrm{D} H(u, v, \alpha)=\left(E u+\alpha, v, u-h^{-1}(\alpha)\right)
$$

and observe that by (6.2),

$$
-\mathrm{D} H(u, v, \alpha) R(u, v, \alpha)=\left(u-h^{-1}(\alpha)\right)(\alpha-h(u)) \geqslant \frac{1}{E}(\alpha-h(u))^{2},
$$

which implies (H5).

We next check the entropy consistency between the systems (6.1) and (6.6). First we observe that

$$
q(u, v):=Q(M(u, v))=(h(u)+E u) v=\sigma(u) v .
$$

Also, we have

$$
\eta(u, v):=H(M(u, v))=\frac{1}{2} v^{2}+\Sigma(u)+k(u),
$$

where

$$
k(u):=\frac{1}{2} E u^{2}+h(u) u-\int_{0}^{h(u)} h^{-1}(\xi) d \xi-\int_{0}^{u} \sigma(\xi) d \xi .
$$

From (6.7) it follows that $k(0)=0, k^{\prime}(u)=0$ for all $u \in \mathbb{R}$, and hence

$$
\eta(u, v)=\frac{1}{2} v^{2}+\Sigma(u) .
$$

Then, (6.5), (6.13), and (6.12) imply (H6). Next, notice that

$$
\left|(u, v, \alpha)^{\top}-M(u, v)\right|=|\alpha-h(u)|,
$$

and hence (4.2), (4.3), and (6.11) imply (H7) with $\nu=\frac{1}{E}$. 
Finally, we observe that

$$
\begin{aligned}
& {[\mathrm{D} H(u, v, \alpha)-\mathrm{D} H(M(\bar{u}, \bar{v}))][G(u, v, \alpha)-G(M(\bar{u}, \bar{v}))] } \\
= & (v-\bar{v})\left(g_{2}(u, v)-g_{2}(\bar{u}, \bar{v})\right) \\
= & {[\mathrm{D} \eta(u, v)-\mathrm{D} \eta(\bar{u}, \bar{v})][g(u, v)-g(\bar{u}, \bar{v})] }
\end{aligned}
$$

for each $(u, v, \alpha)^{\top}, M(\bar{u}, \bar{v}) \in \mathbb{R}^{3}$. Then, if the source $g(u, v)$ satisfies (6.3), then (6.14) implies (H8). If, on the other hand, $g(u, v)$ satisfies (6.4), then (6.14) implies (H9).

Thus, if $\left\{\left(u^{\varepsilon}, v^{\varepsilon}, \alpha^{\varepsilon}\right)\right\}$ is a uniformly bounded family of weak solutions, one may apply Theorem 3.3 to establish convergence before formation of shocks. If such a priori information is not available, then, in addition to (6.2)-(6.4), we require that

$$
\left|\sigma^{\prime \prime}(u)\right| \leqslant K, \quad u \in \mathbb{R} .
$$

In that case, from (6.10) and (6.15) it follows that (3.1) and (3.2) hold and therefore one may apply theorems 3.1 and 3.2 (depending on the type of source term).

REMARK 6.1. Replacing (6.3) with the weakly dissipative condition

$$
\left(g_{2}(v)-g_{2}(\bar{v})\right)(v-\bar{v}) \leqslant-c|v-\bar{v}|^{2}, \quad \forall v, \bar{v} \in \mathbb{R},
$$

the relaxation system falls into the framework of $[16,26]$, which provides global smooth solutions for small initial data. The case of Lipschitz source terms can also be handled following a line of argument similar to that in $[16,26]$. Note that the same follows for the combustion model presented below, which has a Lipschitz source term.

\section{Application to combustion}

The governing equations for a chemical reaction from unburnt gases to burnt gases in certain physical regimes read [8]:

$$
\begin{aligned}
& \partial_{t} v-\partial_{x} u=0, \\
& \partial_{t} u+\partial_{x}(P(v, s, Z))=0, \\
& \partial_{t}\left(E(v, s, Z)+\frac{1}{2} u^{2}+q Z\right)_{t}+\partial_{x}(u P(v, s, Z))=r, \\
& \partial_{t} Z+K \varphi(\Theta(v, s, Z)) Z=0 .
\end{aligned}
$$

The state of the gas is characterized by the macroscopic variables: the specific volume $v(x, t)$, the velocity field $u(x, t)$, the entropy $s(x, t)$, and the mass fraction of the reactant $Z(x, t)$, whereas the physical properties of the material are reflected through appropriate constitutive relations which relate the pressure $P(v, s, Z)$ and internal energy $E(v, s, Z)$ with the macroscopic variables. Here, and in what follows, $q$ represents the difference in the heats between the reactant and the product, $K$ denotes the rate of the reactant, whereas $\varphi(\theta) \geqslant 0$ is the reaction function. The function $r(x, t)$ represents a source term (additional radiating heat density).

Isentropic combustion. In this section we address the problem of relaxation to the isentropic combustion model

$$
\left(\begin{array}{l}
v \\
u \\
Z
\end{array}\right)_{t}+\left(\begin{array}{c}
-u \\
P(v, Z) \\
0
\end{array}\right)_{x}=\left(\begin{array}{c}
0 \\
0 \\
-K \varphi(\Theta(v, Z))
\end{array}\right)
$$


that arises naturally from (7.1) by externally regulating $r$ to ensure $s=s_{0}$ [9], in which case we suppress variable $s$ and use the notation

$$
P(v, Z):=P\left(v, s_{0}, Z\right), \quad \Theta(v, Z):=\Theta\left(v, s_{0}, Z\right) .
$$

Motivation for assumptions. Our main objective is to find a proper extended system associated with the system (7.2) that models isentropic processes with specific volume $v$ away from both zero and vacuum, that is, when $v$ has upper and lower bounds,

$$
v_{0} \leqslant v \leqslant V_{0} \text { for some } v_{0}, V_{0} \in(0, \infty) \text {. }
$$

For the rest of the paper we assume that the a priori bound (7.4) holds.

The physics of (isentropic) thermodynamical processes determined by the equations (7.1) and compatible with the Clausius-Duhem inequality require the choice of the pressure $P(v, Z)$ and temperature $\Theta(v, Z)$ which are compatible with the following properties: for $v \in\left[v_{0}, V_{0}\right], s=s_{0}$, and $Z \in[0,1]$,

$$
P(v, z)=-\partial_{v} E\left(v, s_{0}, Z\right)>0, \quad \Theta(v, Z)=\partial_{s} E\left(v, s_{0}, Z\right)>0
$$

for some (appropriate) energy function

$$
E(v, s, Z)>0 \quad \text { with } \quad E_{Z}\left(v, s_{0}, Z\right)>0 .
$$

We remark that such a function $E(v, s, Z)$ is known to exist for the system (7.1) as long as $v, s$ have lower and upper bounds [8].

For technical convenience, outside of the interval (7.4), we redefine the constitutive law $E\left(v, s_{0}, Z\right)$ ensuring that the functions $P(v, Z), \Theta(v, Z)$ are defined for all $v \in \mathbb{R}$, $Z \in[0,1]$ with bounded derivatives as indicated below.

\section{Conditions on $P, \Theta$.}

(a1) Motivated by the physical property $\partial_{v} P<0$ we assume that

$$
0<\gamma<-\partial_{v} P(v, Z)<\Gamma, \quad v \in \mathbb{R}, Z \in[0,1] .
$$

(a2) There exists $\bar{C}>0$ such that

$$
\left|\int_{0}^{v} P_{Z Z}(\tau, Z) d \tau\right|<\bar{C}, \quad\left|\partial_{Z} P(v, Z)\right|<\bar{C}, \quad v \in \mathbb{R}, Z \in[0,1] .
$$

(a3) The composition $\varphi \circ \Theta$ of the rate and constitutive temperature functions satisfies for some $L>0$

$$
|\varphi(\Theta(v, Z))-\varphi(\Theta(\bar{v}, \bar{Z}))| \leqslant L|(v, Z)-(\bar{v}, \bar{Z})|
$$

for all $(v, Z),(\bar{v}, \bar{Z}) \in \mathbb{R} \times[0,1]$. form:

Under (a1)-(a3) the system (7.2) admits an entropy-entropy flux pair $\bar{\eta}, \bar{q}$ of the

$$
\begin{aligned}
\bar{\eta}(v, u, Z) & =\frac{1}{2} u^{2}-\left(\int_{0}^{v} P(\tau, Z) d \tau\right)+B(Z), \\
\bar{q}(v, u, Z) & =P(v, Z) u
\end{aligned}
$$


where $B(Z)$ is an arbitrary function.

Relaxation via approximation of pressure. In the spirit of the example for the elasticity system (6.1) we define

$$
h(v, Z):=-P(v, Z)-E v \quad \text { with } \quad E>\Gamma .
$$

We now approximate the pressure $P(v, Z)$ by the linear combination $-(\alpha+E v)$. This leads to the extended system

$$
\left(\begin{array}{c}
v \\
u \\
Z \\
\alpha
\end{array}\right)_{t}-\left(\begin{array}{c}
u \\
\alpha+E v \\
0 \\
0
\end{array}\right)_{x}=\frac{1}{\varepsilon} R(v, u, Z, \alpha)+G(v, u, Z, \alpha)
$$

where

$$
\begin{aligned}
& R(v, u, Z, \alpha)=[0,0,0, h(v, Z)-\alpha]^{\top} \\
& G(v, u, Z, \alpha)=[0,0,-K \varphi(\Theta) Z, 0]^{\top} .
\end{aligned}
$$

Note that as $\varepsilon \rightarrow 0, \alpha$ tends to its equilibrium state $\alpha_{e q}=h\left(v_{e q}, Z_{e q}\right)$. Then

$$
\alpha_{e q}+E v_{e q}=-P\left(v_{e q}, Z_{e q}\right),
$$

and hence $\left(v_{e q}, u_{e q}, Z_{e q}\right)$ solves (7.2). This motivates the parametrization of the manifold of Maxwellians $\mathcal{M}$ by

$$
M(v, u, Z)=[v, u, Z, h(v, Z)]^{\top},
$$

which yields (H1). Next, we compute

$$
\mathrm{D} R(v, u, Z, \alpha)=\left[\begin{array}{cccc}
0 & 0 & 0 & 0 \\
0 & 0 & 0 & 0 \\
0 & 0 & 0 & 0 \\
h_{v}(v, Z) & 0 & h_{Z}(v, Z) & -1
\end{array}\right],
$$

from which we conclude

$$
\operatorname{dim} \mathcal{N}(\mathrm{D} R(M(v, u, Z)))=3, \quad \operatorname{dim} \mathcal{R}(\mathrm{D} R(M(v, u, Z)))=1,
$$

which verifies (H2) for $n=3, N=4$. We choose the projection matrix

$$
\mathbb{P}=\left[\begin{array}{llll}
1 & 0 & 0 & 0 \\
0 & 1 & 0 & 0 \\
0 & 0 & 1 & 0
\end{array}\right],
$$

for which $\mathbb{P} M(v, u, Z)=(v, u, Z)^{\top}$ and $\mathbb{P} R(v, u, Z, \alpha)=\mathbf{0}$, and hence (H3) holds.

Entropy of the extended system. We next specify the entropy-entropy flux pair of the relaxation system (7.12). By (7.7)

$$
0<E-\Gamma<-h_{v}(v, Z)<E-\gamma .
$$


Hence there exists $j(\alpha, Z): \mathbb{R} \times[0,1] \rightarrow \mathbb{R}$ such that

$$
j(h(v, Z), Z)=v, \quad h(j(\alpha, Z), Z)=\alpha
$$

for all $v, \alpha \in \mathbb{R}, Z \in[0,1]$. Thus, we define

$$
\begin{aligned}
H(v, u, Z, \alpha) & :=\frac{u^{2}}{2}-\int_{h(0, Z)}^{\alpha} j(\xi, Z) d \xi+\alpha v+\frac{E v^{2}}{2}+B(Z), \\
Q(u, v, \alpha) & :=-(\alpha+E v) u
\end{aligned}
$$

with $B(Z)$ an arbitrary function such that

$$
B^{\prime \prime}(Z)>m>0, \quad Z \in[0,1],
$$

where the constant $m>0$ is to be specified.

It is easy to check that $H, Q$ is the entropy-entropy flux pair for (7.12). To show that $H(U)$ is strictly convex, however, is less trivial and therefore, for the convenience of a reader, we provide detailed calculations. Recalling that $E>\Gamma>\gamma$ we rewrite $H(v, u, Z, \alpha)$ as follows:

$$
H(u, v, Z, \alpha)=\left(\frac{u^{2}}{2}+\frac{\gamma v^{2}}{4}+\psi(\alpha, Z)\right)+\frac{(\alpha+\widehat{E} v)^{2}}{2 \widehat{E}},
$$

with

$$
\psi(\alpha, Z):=-\int_{h(0, Z)}^{\alpha} j(\xi, Z) d \xi-\frac{\alpha^{2}}{2 \widehat{E}}+B(Z), \quad \widehat{E}:=E-\frac{\gamma}{2} .
$$

We now show that there exists $\Lambda>0$ such that

$$
\Lambda^{-1} \mathbf{I} \leqslant \mathrm{D}^{2} \psi(\alpha, Z) \leqslant \Lambda \mathbf{I}
$$

by establishing the bounds on the eigenvalues of

$$
\mathrm{D}^{2} \psi(\alpha, Z)=\left[\begin{array}{cc}
-j_{\alpha}(\alpha, Z)-\widehat{E}^{-1} & -j_{Z}(\alpha, Z) \\
-j_{Z}(\alpha, Z) & B^{\prime \prime}(Z)-\partial_{Z Z}\left(\int_{h(0, Z)}^{\alpha} j(\xi, Z) d \xi\right)
\end{array}\right]
$$

Differentiating $(7.15)_{2}$ and recalling (7.11) we get

$$
j_{\alpha}(\alpha, Z)=\frac{1}{h_{v}(j(\alpha, Z), Z)}, \quad j_{Z}(\alpha, Z)=\frac{P_{Z}(j(\alpha, Z), Z)}{h_{v}(j(\alpha, Z), Z)},
$$

and hence, by (7.8) and (7.14),

$$
\frac{1}{E-\gamma} \leqslant-j_{\alpha}(\alpha, Z) \leqslant \frac{1}{E-\Gamma}, \quad\left|j_{Z}(\alpha, Z)\right| \leqslant \frac{\bar{C}}{E-\Gamma} .
$$

Then by $(7.21)_{1}$

$$
\frac{\gamma}{2(E-\gamma) \widehat{E}} \leqslant\left[\mathrm{D}^{2} \psi(\alpha, Z)\right]_{11} \leqslant \frac{\Gamma-\frac{1}{2} \gamma}{(E-\Gamma) \widehat{E}} .
$$


Next, using (7.15) and (7.20) $)_{1,2}$, we compute

$$
\begin{aligned}
& \partial_{Z}\left(\int_{h(0, Z)}^{\alpha} j(\xi, Z) d \xi\right) \\
= & \int_{h(0, Z)}^{\alpha} j_{Z}(\xi, Z) d \xi-\left(j(h(0, Z), Z) h_{Z}(0, Z)\right) \\
= & \int_{h(0, Z)}^{\alpha} P_{Z}(j(\xi, Z), Z) j_{\alpha}(\xi, Z) d \xi=\int_{0}^{j(\alpha, Z)} P_{Z}(\tau, Z) d \tau,
\end{aligned}
$$

and hence

$$
\begin{aligned}
& \partial_{Z Z}\left(\int_{h(0, Z)}^{\alpha} j(\xi, Z) d \xi\right) \\
= & P_{Z}(j(\alpha, Z), Z) j_{Z}(\alpha, Z)+\int_{0}^{j(\alpha, Z)} P_{Z Z}(\tau, Z) d \tau .
\end{aligned}
$$

Then, by (7.8) and $(7.21)_{2}$, we conclude

$$
\left|\partial_{Z Z}\left(\int_{0}^{\alpha} j(\xi, Z) d \xi\right)\right| \leqslant \bar{C}\left(1+\frac{\bar{C}}{E-\Gamma}\right) .
$$

The analysis of the above inequalities motivates us to choose

$$
m:=\widehat{m}+\bar{C}\left(1+\frac{\bar{C}}{E-\Gamma}\right) \text { with } \widehat{m}:=\left[\left(\frac{\bar{C}^{2}}{E-\Gamma}\right)^{2}+1\right] \frac{2(E-\gamma) \widehat{E}}{\gamma},
$$

in which case by (7.16), (7.19), and (7.23) we obtain

$$
0<\widehat{m} \leqslant\left[\mathrm{D}^{2} \psi(\alpha, Z)\right]_{22} \leqslant 2 m
$$

Combining $(7.21)_{2},(7.22)$, and (7.24) we get

$$
1 \leqslant \operatorname{det}\left[\mathrm{D}^{2} \psi(\alpha, Z)\right]=\lambda_{1} \lambda_{2}
$$

where $\lambda_{1}, \lambda_{2} \in \mathbb{R}$ denote the largest and smallest eigenvalues of $\mathrm{D}^{2} \psi$, respectively. Observe that (7.19), (7.22), and (7.24) imply

$$
0<\lambda_{1} \leqslant \Lambda:=\left(2 m+\frac{\Gamma-\frac{\gamma}{2}}{(E-\Gamma) \widehat{E}}+\frac{\bar{C}^{2}}{E-\Gamma}\right)
$$

Then, from (7.25), (7.26) we obtain the estimate (7.18).

Combining (7.17), (7.18) we conclude that for some $\mu, \mu^{\prime}>0$

$$
\mu \mathbf{I} \leqslant \mathrm{D}^{2} H(v, u, Z, \alpha) \leqslant \mu^{\prime} \mathbf{I},
$$

and this yields (H4).

Now, recalling (7.13), (7.15), and $(7.21)_{1}$ we obtain

$$
\begin{aligned}
& -\mathrm{D} H(v, u, Z, \alpha) R(v, u, Z, \alpha) \\
= & -(j(h(v, Z), Z)-j(\alpha, Z))(h(v, Z)-\alpha) \\
= & -\left[\int_{0}^{1} j_{\alpha}(\operatorname{sh}(v, Z)+(1-s) \alpha, Z) d s\right](h(v, Z)-\alpha)^{2} \\
\geqslant & \frac{1}{E-\gamma}(h(v, Z)-\alpha)^{2}=\frac{1}{E-\gamma}\left|M(v, u, Z)-(v, u, Z, \alpha)^{\top}\right|^{2},
\end{aligned}
$$


which implies that the entropy $H$ satisfies hypotheses (H5) and (H7).

Next, we observe that $(7.11),(7.15)$, and $(7.20)_{1}$ imply

$$
\int_{h(0, Z)}^{h(v, Z)} j(\xi, Z) d \xi=\int_{0}^{v} h_{v}(\tau, Z) \tau d \tau=h(v, Z) v+\frac{E v^{2}}{2}+\int_{0}^{v} P(v, Z) d \tau .
$$

Thus, the entropy pair $H, Q$ restricted to the equilibrium manifold satisfies

$$
\begin{aligned}
& \eta(v, u, Z):=H(M(v, u, Z))=\frac{u^{2}}{2}-\int_{0}^{v} P(v, Z) d \tau+B(Z), \\
& q(v, u, Z):=Q(M(v, u, Z))=-(h(v, Z)+E v) u=P(v, Z) u .
\end{aligned}
$$

Then, (7.28) together with (7.10) yields (H6).

Consider an arbitrary compact set $\mathcal{A} \subset \mathbb{R} \times \mathbb{R} \times[0,1] \times \mathbb{R}$. Then, by (7.9), for all

$$
(v, u, Z, \alpha) \in \mathbb{R} \times \mathbb{R} \times[0,1] \times \mathbb{R}, \quad(\bar{v}, \bar{u}, \bar{Z}, \bar{\alpha}) \in \mathcal{A}
$$

we have

$$
\begin{aligned}
& |G(v, u, Z, \alpha)-G(\bar{v}, \bar{u}, \bar{Z}, \bar{\alpha})| \\
= & |K \varphi(\Theta(v, Z)) Z-K \varphi(\Theta(\bar{v}, \bar{Z})) \bar{Z}| \\
\leqslant & |K|(|Z||\varphi(\Theta(v, Z))-\varphi(\Theta(\bar{v}, \bar{Z}))|+|\varphi(\Theta(\bar{v}, \bar{Z}))||Z-\bar{Z}|) \\
\leqslant & \left(L+L_{\mathcal{A}}\right)|K||(v, u, Z, \alpha)-(\bar{v}, \bar{u}, \bar{Z}, \bar{\alpha})|,
\end{aligned}
$$

where $L_{\mathcal{A}}>0$ denotes a constant for which, in view of (7.9),

$$
|\varphi(\Theta(\bar{v}, \bar{Z}))| \leqslant L_{\mathcal{A}}, \quad(\bar{v}, \bar{u}, \bar{Z}, \bar{\alpha}) \in \mathcal{A} .
$$

The estimate (7.29) implies that the source $G$ satisfies the hypothesis (H9) on $\mathbb{R} \times \mathbb{R} \times[0,1] \times \mathbb{R}$, the state space of $(7.12)$ with initial data such that $0 \leqslant Z(\cdot, 0) \leqslant 1$. Thus, if the family $\left\{\left(v^{\varepsilon}, u^{\varepsilon}, Z^{\varepsilon}, \alpha^{\varepsilon}\right)\right\}$ is uniformly bounded, one may apply Theorem 3.3 to establish convergence before the formation of shocks. If such a priori information is not available, then, in addition to $(a 1)-(a 3)$, we require that

$$
\left|\mathrm{D}^{2} P(v, Z)\right| \leqslant K, \quad\left|B^{\prime \prime \prime}(Z)\right|<K, \quad v \in \mathbb{R}, Z \in[0,1] \subset \mathbb{R} .
$$

In that case, from (7.27) and (7.30) it follows that (3.1) and (3.2) hold and therefore one may apply Theorem 3.2.

8. General framework for symmetric hyperbolic systems, $d=1$

In this section we present a general strategy indicating how, starting from a symmetric hyperbolic system, one can construct an extended relaxation system.

Consider the hyperbolic balance law

$$
\partial_{t} u+\partial_{x} f(u)=g(u), \quad u, f(u), g(u) \in \mathbb{R}^{n}
$$

such that:

- The flux $f(u)$ has symmetric $\mathrm{D}_{u} f(u)$. Thus,

$$
f(u)=\mathrm{D} \Phi^{\top}(u) \quad \text { for some } \quad \Phi(u): \mathbb{R}^{n} \rightarrow \mathbb{R} .
$$


- $\Phi$ is convex and for some $\Gamma, \gamma>0$ such that

$$
0<\gamma<\mathrm{D}^{2} \Phi(u)<\Gamma, \quad u \in \mathbb{R}^{n} .
$$

- For each compact $\mathcal{A} \subset \mathbb{R}^{n}$ there exists $L_{\mathcal{A}}>0$ such that

$$
|g(u)-g(\bar{u})| \leqslant L_{\mathcal{A}}|u-\bar{u}|, \quad u \in \mathbb{R}^{n}, \bar{u} \in \mathcal{A} .
$$

By (h1) the system (8.1) admits entropy-entropy flux pair

$$
\bar{\eta}(u)=\Phi(u), \quad \bar{q}(u)=\frac{1}{2}|\mathrm{D} \Phi(u)|^{2} .
$$

Relaxation via flux approximation. Next, we approximate the flux $f(u)$ by the combination $\alpha+\mathrm{DE}^{\top}(u)$, where $\alpha \in \mathbb{R}^{n}$ is a new vector variable and $\mathcal{E}(u): \mathbb{R}^{n} \rightarrow \mathbb{R}$ is a convex function such that for some $E, \delta>0$ there holds

$$
(E+\delta) \mathbf{I} \geqslant \mathrm{D}_{u}^{2} \mathcal{E}(u) \geqslant E \mathbf{I}, \quad E>\Gamma>\gamma>\delta>0 .
$$

This leads to the relaxation system for variables $u, \alpha \in \mathbb{R}^{n}$,

$$
\left(\begin{array}{l}
u \\
\alpha
\end{array}\right)_{t}+\left(\begin{array}{c}
\alpha+\mathrm{DE}^{\top}(u) \\
0
\end{array}\right)_{x}=\frac{1}{\varepsilon} R(u, \alpha)+G(u, \alpha),
$$

with

$$
R(u, \alpha)=[0, h(u)-\alpha]^{\top}, \quad G(u, \alpha)=[g(u), 0]^{\top} .
$$

We now define

$$
\Sigma(u):=\mathcal{E}(u)-\Phi(u), \quad h(u):=-\mathrm{D}_{u} \Sigma^{\top}(u)=f(u)-\mathrm{D}_{u} \mathcal{E}^{\top}(u) .
$$

Then, by (h2) and (h4), we have

$$
\mathrm{D}_{u}^{2} \mathcal{E} \geqslant E \mathbf{I}>(E+\delta-\gamma) \mathbf{I}>\mathrm{D}_{u}^{2} \Sigma>(E-\Gamma) \mathbf{I} .
$$

The mapping

$$
\mathrm{D}_{u} \Sigma: \mathbb{R}^{n} \rightarrow \mathbb{R}^{n} \quad \text { is onto, }
$$

as implied by the following lemma (cf. Zeidler [27]).

Lemma 8.1. Suppose $V(u): \mathbb{R}^{n} \rightarrow \mathbb{R}^{n}$ is a $C^{1}$-mapping such that

$$
\mathrm{D} V(u): \mathbb{R}^{n} \rightarrow \mathbb{R}^{n} \text { is invertible for all } u \in \mathbb{R}^{n},
$$

and the map $V(u)$ is coercive, that is, $V(u)^{\top} u>c|u|^{2}, u \in \mathbb{R}^{n}$ for some fixed constant $c>0$. Then, $V$ must be surjective and covers all of $\mathbb{R}^{n}$.

Observe that as $\varepsilon \rightarrow 0, \alpha$ tends to its equilibrium state $\alpha_{e q}=h\left(u_{e q}\right)$ in which case the corresponding equilibrium state $u_{e q}$ satisfies (8.1). This suggests the parametrization of the manifold of Maxwellians by

$$
M(u)=[u, h(u)]^{\top},
$$


which yields (H1). Next, observe that

$$
\operatorname{dim} \mathcal{N}(\mathrm{D} R(M(u)))=n, \quad \operatorname{dim} \mathcal{R}(\mathrm{D} R(M(u)))=n,
$$

which verifies (H2) with $N=2 n$. The structure of (8.1) and (8.2) suggests the choice of the projection matrix

$$
\mathbb{P}=[\mathbf{I}, \mathbf{0}]: \mathbb{R}^{2 n} \rightarrow \mathbb{R}^{n} \quad \text { for which } \quad \mathbb{P} M(u)=u, \mathbb{P} R(u, \alpha)=\mathbf{0},
$$

which implies (H3).

To construct the entropy-entropy flux pair for the relaxation system (8.2) we exploit the ideas of the analysis of A. Tzavaras [24]. By (8.5) and (8.6) the map D $\Sigma$ is bijective. This motivates the definition of

$$
j(\alpha): \mathbb{R}^{n} \rightarrow \mathbb{R}^{n} \quad \text { by } \quad j(\alpha)^{\top}=-\left(\mathrm{D}_{u} \Sigma\right)^{-1}(-\alpha), \quad \alpha \in \mathbb{R}^{n} .
$$

Then, by the inverse mapping theorem, $\mathrm{D}_{\alpha} j(\alpha)$ is symmetric and hence there exists $J(\alpha): \mathbb{R}^{n} \rightarrow \mathbb{R}$ such that

$$
\begin{aligned}
& \mathrm{D}_{\alpha} J(\alpha)=j(\alpha)^{\top}=-(\mathrm{D} \Sigma)^{-1}(-\alpha), \\
& \mathrm{D}_{\alpha}^{2} J(\alpha)=\left[\mathrm{D}_{u}^{2} \Sigma\left(-\mathrm{D}_{\alpha} J^{\top}(\alpha)\right)\right]^{-1}=\left[\mathrm{D}_{u}^{2} \Sigma(-j(\alpha))\right]^{-1} .
\end{aligned}
$$

Furthermore, by (8.5) we obtain that $J(\alpha)$ is uniformly convex with

$$
(E+\Gamma)^{-1} \mathbf{I}>\mathrm{D}_{\alpha}^{2} J(\alpha)>(E+\delta-\gamma)^{-1} \mathbf{I} .
$$

We next define

$$
\begin{aligned}
H(u, \alpha) & =\mathcal{E}(u)+\alpha^{\top} u+J(\alpha), \\
Q(u, \alpha) & =\frac{1}{2}\left|\alpha+\mathrm{DE}^{\top}(u)\right|^{2} .
\end{aligned}
$$

It is easy to verify that $H, Q$ is the entropy-entropy flux pair for the system (8.2). To show that $H(u, \alpha)$ is strictly convex, we compute the Hessian

$$
\mathrm{D}^{2} H(u, \alpha)=\left[\begin{array}{cc}
\mathrm{D}_{u}^{2} \mathcal{E}(u) & \mathbf{I} \\
\mathbf{I} & \mathrm{D}_{\alpha}^{2} J(\alpha)
\end{array}\right]
$$

and write

$$
(u, \alpha)^{\top}\left[\mathrm{D}^{2} H(u, \alpha)\right](u, \alpha)=u^{\top}\left[\mathrm{D}^{2} \mathcal{E}(u)\right] u+2 \alpha^{\top} u+\alpha^{\top}\left[\mathrm{D}^{2} J(\alpha)\right] \alpha .
$$

Then, recalling (h4), (8.5), and (8.9), we get the estimates

$$
(u, \alpha)^{\top}\left[\mathrm{D}^{2} H(u, \alpha)\right](u, \alpha)>\frac{1}{2}(\gamma-\delta)|u|^{2}+\frac{\frac{1}{2}(\gamma-\delta)|\alpha|^{2}}{\left(E+\frac{1}{2}(\delta-\gamma)\right)(E+\delta-\gamma)}
$$

and

$$
(u, \alpha)^{\top}\left[\mathrm{D}^{2} H(u, \alpha)\right](u, \alpha) \leqslant(E+\delta+1)|u|^{2}+\left((E-\Gamma)^{-1}+1\right)|\alpha|^{2} .
$$


The above inequalities and the fact that $\gamma>\delta$ imply that there exist $\mu, \mu^{\prime}>0$ such that

$$
\mu^{\prime} \mathbf{I} \leqslant \mathrm{D}^{2} H(u, \alpha) \leqslant \mu \mathbf{I}, \quad(u, \alpha) \in \mathbb{R}^{n+n},
$$

and hence we conclude that the pair $H, Q$ satisfies (H4).

Next, we compute

$$
\mathrm{D} H(u, \alpha)=\left[\mathrm{D}_{u} \mathcal{E}(u)+\alpha^{\top}, u^{\top}+\mathrm{D}_{\alpha} J(\alpha)\right]
$$

and observe that, by $(8.4)_{1}$ and (8.7),

$$
-j(h(u))=(\mathrm{D} \Sigma)^{-1}(-h(u))=(\mathrm{D} \Sigma)^{-1}\left(\mathrm{D} \Sigma^{\top}(u)\right)=u .
$$

Hence recalling $(8.3)_{1},(8.4),(8.8)_{1}$, and (8.9) we obtain

$$
\begin{aligned}
& -\mathrm{DH}(u, \alpha) R(u, \alpha) \\
= & \left(u^{\top}+\mathrm{D}_{\alpha} J(\alpha)\right)(\alpha-h(u)) \\
= & (j(\alpha)-j(h(u)))^{\top}(\alpha-h(u)) \\
= & (\alpha-h(u))^{\top}\left[\int_{0}^{1} \mathrm{D}^{2} J(s \alpha+(1-s) h(u)) d s\right](\alpha-h(u)) \\
\geqslant & \frac{1}{(E+\delta-\gamma)}|\alpha-h(u)|^{2}=\frac{1}{(E+\delta-\gamma)}\left|(u, \alpha)^{\top}-M(u)\right|^{2} .
\end{aligned}
$$

The last inequality implies that $H$ satisfies hypotheses (H5) and (H7).

Next, observe that, by (8.4) and (8.10),

$$
\begin{aligned}
\eta(u):=H(M(u)) & =H(u, h(u)) \\
& =\mathcal{E}(u)+h(u)^{\top} u+J(h(u)) \\
& =\Phi(u)+\Sigma(u)-\left[\mathrm{D}_{u} \Sigma(u)\right] u+J(h(u)) .
\end{aligned}
$$

Then, by (8.4), $(8.8)_{1}$, and (8.12),

$$
\mathrm{D}_{u} \eta(u)=\mathrm{D}_{u} \Phi(u)-\left[\mathrm{D}_{u}^{2} \Sigma(u)\right] u+j(h(u))^{\top}\left[-\mathrm{D}_{u}^{2} \Sigma(u)\right]=\mathrm{D}_{u} \Phi(u),
$$

and we conclude

$$
\eta(u)=\Phi(u)+C \quad \text { for some } \quad C \in \mathbb{R} .
$$

Similarly, by (8.4) and (8.10),

$$
q(u):=Q(M(u))=Q(u, h(u))=\frac{1}{2}\left|h(u)+\mathrm{DE}^{\top}(u)\right|^{2}=\frac{1}{2}|\mathrm{D} \Phi(u)|^{2} .
$$

By the discussion in the beginning of the section we conclude that $\eta, q$ defined in (8.13), (8.14) is an entropy-entropy flux pair of (8.1) which implies (H6).

Now, take an arbitrary compact set $\mathcal{C} \subset \mathbb{R}^{n} \times \mathbb{R}^{n}$ and define

$$
\mathcal{A}=\left\{\bar{u} \in \mathbb{R}^{n}: \text { for some } \bar{\alpha} \in \mathbb{R}^{n}(\bar{u}, \bar{\alpha}) \in \mathcal{C}\right\},
$$


which is compact as well. Then, by (h3) for all $(u, \alpha) \in \mathbb{R}^{n+n},(\bar{u}, \bar{\alpha}) \in \mathcal{C}$,

$$
|G(u, \alpha)-G(\bar{u}, \bar{\alpha})|=|g(u)-g(\bar{u})| \leqslant L_{\mathcal{A}}|(u, \alpha)-(\bar{u}, \bar{\alpha})| .
$$

The above estimate shows that $G(u, \alpha)$ satisfies (H9). Thus, the relaxation system (8.2) satisfies (H1)-(H7), (H9). Thus, if $\left\{u^{\varepsilon}\right\}$ is a uniformly bounded family of weak solutions, one may apply Theorem 3.3 to establish convergence. If such a priori information is not available, then, in addition to (h1)-(h4), we require that

$$
\left|\mathrm{D}^{3} \Phi(u)\right| \leqslant K, \quad u \in \mathbb{R}^{n} .
$$

In that case, from (8.11) and (8.15) it follows that (3.1) and (3.2) hold and therefore one may apply Theorem 3.2 to establish convergence in the smooth regime.

Acknowledgments. The authors thank Thanos Tzavaras for several fruitful conversations and suggestions during the course of this investigation. A.M. thanks Robin Young for advice and helpful comments regarding the combustion model. K.T. acknowledges the support by the National Science foundation under the grant DMS1211519 and the support by the Simons Foundation under the grant \#267399.

\section{REFERENCES}

[1] Y. Brenier, Convergence of the Vlasov-Poisson system to the incompressible Euler equations, Commun. Part. Diff. Eqs., 25, 737-754, 2000.

[2] Y. Brenier, R. Natalini, and M. Puel, On the relaxation approximation of the incompressible Navier-Stokes equations, Proc. Amer. Math. Soc., 132, 1021-1028, 2004.

[3] F. Berthelin and A. Vasseur, From kinetic equations to multidimensional isentropic gas dynamics before shocks, SIAM J. Math. Anal., 36, 1807-1835, 2005.

[4] F. Berthelin, A. Vasseur, and A. Tzavaras, From discrete velocity Boltzmann equations to gas dynamics before shocks, J. Stat. Phys., 135, 151-173, 2009.

[5] R. Caflish and G. Papanicolaou, The fluid-dynamical limit of a nonlinear model Boltzmann equation, Commun. Pure Appl. Appl., 32, 589-616, 1979.

[6] G.Q. Chen, C.D. Levermore, and T.P. Liu, Hyperbolic conservation laws with stiff relaxation, Lett. Math. Phys., 22(1), 63-80, 1991.

[7] G.Q. Chen, D. Hoff, and K. Trivisa, On the Navier-Stokes equations for exothermically, reacting, compressible fluids, Acta Math. Appl. Sinica, 18, 15-36, 2002.

[8] G.Q. Chen, D. Hoff, and K. Trivisa, Global solutions to a model for exothermically reacting, compressible flows with large discontinuous initial data, Arch. Ratio. Mech. Anal., 166, 321-358, 2003.

[9] C.M. Dafermos, The second law of thermodynamics and stability, Arch. Ratio. Mech. Anal., 70, 167-179, 1979.

[10] C.M. Dafermos, Quasilinear hyperbolic systems with involutions, Arch. Ratio. Mech. Anal., 94, 373-389, 1986.

[11] C.M. Dafermos, Hyperbolic systems of balance laws with weak dissipation, J. Hyperbolic Diff. Eqs., 3(3), 505-527, 2006.

[12] C.M. Dafermos, Hyperbolic Conservation Laws in Continuum Physics, Third Edition, Grundlehren der Mathematischen Wissenschaften, Springer-Verlag, Berlin, 325, 2010.

[13] R. DiPerna, Uniqueness of solutions to hyperbolic conservation laws, Indiana U. Math. J., 28, 137-188, 1979.

[14] P. Goncalves, C. Landim, and C. Toninelli, Hydrodynamic limit for a particle system with degenerate rates, Ann. Inst. Henri Poincaré, Probab. Stat., 45(4), 887-909, 2009.

[15] S. Jin and M. Katsoulakis, Hyperbolic systems with supercharacteristic relaxations and roll waves, SIAM J. Appl. Math., 61(1), 273-292, 2000.

[16] B. Hanouzet and R. Natalini, Global existence of smooth solutions for partially dissipative hyperbolic systems with a convex entropy, Arch. Ratio. Mech. Anal., 169, 89-117, 2003.

[17] T. Karper, A. Mellet, and K. Trivisa, Hydrodynamic limit of the kinetic Cucker-Smale flocking model, submitted, 2013.

[18] C. Lattanzio and A.E. Tzavaras, Structural properties of stress relaxation and convergence from viscoelasticity to polyconvex elastodynamics, Arch. Ratio. Mech. Anal., 180, 449-492, 2006. 
[19] A. Miroshnikov and A. Tzavaras, A variational approximation scheme for radial polyconvex elasticity that preserves the positivity of Jacobians, Commun. Math. Sci., 10(1), 87-115, 2012.

[20] A. Miroshnikov and A. Tzavaras, Convergence of variational approximation schemes for elastodynamics with polyconvex energy, Z. Anal. Anwend., 33(1), 43-64, 2014.

[21] M. Mourragui, Comportement hydrodynamique et entropie relative des processus de sauts, de naissances et de morts, Ann. Inst. H. Poincaré Probab. Statist., 32, 361-385, 1996.

[22] A. Mellet and A. Vasseur, Asymptotic analysis for a Vlasov-Fokker-Planck compressible Navier-Stokes systems of equations, Commun. Math. Phys., 281, 573-596, 2008.

[23] A. Tzavaras, Relative entropy in hyperbolic relaxation, Commun. Math. Sci., 3(2), 119-132, 2005.

[24] A. Tzavaras, A relaxation theory with polyconvex entropy function converging to elastodynamics, preprint, 2006.

[25] H.T. Yau, Relative entropy and hydrodynamics of Ginzburg-Landau models, Lett. Math. Phys., 22(1), 63-80, 1991.

[26] W.A. Yong, Entropy and global existence for hyperbolic balance laws, Arch. Ratio. Mech. Anal., 172, 247-266, 2004.

[27] E. Zeidler, Nonlinear Functional Analysis and its Applications, Part I., Springer, 1986. 